\title{
Les organisations professionnelles apicoles dans la région de Fès-Meknès au Maroc
}

\author{
El Hassania Mohssine ${ }^{1}$, Salma Bakhchou ${ }^{1}$ et Jean-François Odoux ${ }^{2,3, *}$ \\ ${ }^{1}$ IAV Hassan II, Département Productions et Biotechnologies Animales, Rabat, Maroc \\ 2 INRAE-UNICAEN EVA-UMR 950, Écologie des prairies, Université de Caen, Caen, France \\ 3 INRAE-APIS UE1255, Le Magneraud, 17700 Surgères, France
}

\begin{abstract}
Résumé - La filière apicole est largement soutenue par l'État au Maroc. La région de Fès-Meknès a choisi de positionner l'apiculture comme un levier de développement rural. À travers un recensement auprès des administrations concernées, puis d'enquêtes auprès de 44 organisations professionnelles apicoles, notre étude propose une approche s'inscrivant dans un diagnostic de filière régionale. Les données recueillies révèlent l'importance des coopératives dans les formes collectives de production apicole et l'implication de jeunes ruraux et de femmes dans les processus de décision. Les techniques apicoles sont hétérogènes, traduisant une adaptation des méthodes traditionnelles aux techniques modernes, mais avec certaines pratiques, notamment sanitaires, qui restent empiriques. La formation initiale des personnes est un atout aussi bien pour la structuration de la filière que du point de vue de la maitrise du cheptel. Les ressources florales sont variées et continues au niveau de cette grande région, permettant d'imaginer des transhumances pour récolter des miels typiques dans le respect des potentiels disponibles. Peu équipés en matériel inox, les apiculteurs restent demandeurs en termes d'équipements de miellerie. La production modeste est largement écoulée au détail et le conditionnement reste à améliorer pour une bonne reconnaissance du produit. La participation des apiculteurs aux salons régionaux contribue favorablement à une image identitaire qui devra être cultivée.
\end{abstract}

Mots clés : Maroc / coopérative / miel / apiculture / développement rural

\begin{abstract}
Beekeeping professional organisations in the Fès-Meknès Region in Morocco. Moroccan beekeeping is widely supported by the Government. The Region of Fès-Meknès chose to position beekeeping as a lever for rural development. Through a census with regional authorities then interviews with 44 beekeeping groups, our study proposes an approach that is part of a sector diagnosis. The data collected reveal the importance of cooperatives in collective forms of beekeeping production, and the involvement of rural youth and women in decision-making processes. The heterogeneous beekeeping practices reflect an adaptation of traditional methods to modern techniques, but certain practices, in particular bee health, remain empirical. The initial training of people is an asset for the sector structuration and can also allow a good livestock management by breeders. We emphasized the diversity of honeys to highlight the region's beekeeping potential through varied and continuous floral resources allowing hive moving in order to harvest typical honeys in accordance with the available melliferous potential. Little equipped with stainless steel tools, beekeepers remain demanding in terms of honey houses' equipment. The modest production is largely sold at retail and the packaging is still to be improved for an appropriate recognition of the product. Participation of beekeepers in some shows contributes favourably to building a regional identity image.
\end{abstract}

Keywords: Morocco / cooperatives / honey / apiculture / rural development

\footnotetext{
*Auteur de correspondance : jean-francois.odoux@inrae.fr
} 


\section{Introduction}

L'apiculture est un levier de développement rural accessible à des acteurs qui ne possèdent pas de terres et qui n'exige que des moyens relativement limités. À ce titre, les pouvoirs publics considèrent cette activité comme stratégique, à la fois d'un point de vue socio-économique et agroécologique. Au Maroc en particulier, l'apiculture tient traditionnellement une place importante dans la société. Le corpus documentaire concernant l'apiculture et les miels marocains est abondant (e.g. Louveaux et Abed, 1984; Aazza et al., 2014; Chakir et al., 2016; Petretto et al., 2017). Pourtant, malgré des efforts importants de l'État pour moderniser la filière, son développement reste bien limité, en particulier du fait de difficultés techniques (Moujanni et al., 2017). Le travail présenté ici propose d'élaborer un diagnostic de la filière miel au niveau d'une région pour tenter d'évaluer l'impact des politiques publiques de l'État dans les projets de développement apicole, d'identifier les types de miel et d'explorer les potentialités mellifères.

Depuis 2008, le Plan Maroc Vert (PMV) soutient l'agriculture à haute valeur ajoutée mais aussi, dans le cadre de son second pilier, l'accompagnement solidaire de la petite agriculture en favorisant l'organisation collective des petites exploitations (MAPM, 2015). Il a permis une augmentation de la production agricole mais la dynamique de regroupement doit être encore mieux accompagnée (Stührenberg, 2016). L'apiculture est encore en pleine transition au Maroc où la ruche à cadres moderne a progressivement pénétré toutes les régions, faisant se juxtaposer plusieurs modèles sociotechniques (Simenel et al., 2015). Afin d'atteindre l'objectif de production de l'État de 16000 tonnes de miel en 2020 (MAPM, 2015), des projets de développement ont vu le jour dans différentes régions du Maroc, octroyant des ruches et du matériel apicole en quantité et dispensant des formations aux apiculteurs.

Parmi les 12 régions du Maroc, Fès-Meknès ne figure pas parmi les grandes zones apicoles du pays; les miels et les potentiels mellifères de ses diverses provinces (Fig. 1) sont peu documentés. Le miel produit représente moins de $400 \mathrm{t} / \mathrm{an}$, soit environ $5 \%$ de la production nationale (Chambre-Agriculture, 2019; Joual, 2019), loin derrière les anciennes régions du Gharb, Doukkala, Tadla ou Tafilalet (Kabbaj, 2008). Cependant, à l'instar d'autres régions, l'INDH (Initiative nationale pour le développement humain), la DRA (Direction régionale de l'agriculture) et les DPA (Direction provinciale de l'agriculture) y financent, en particulier depuis 2011, des projets de développement apicoles; la filière y connaît aujourd'hui un développement sensible. L'apiculture ne figurant pas dans les statistiques générales, la collecte d'informations dans ce secteur économique est une entreprise très difficile. Les données disponibles indiquent un niveau de production nationale variable de 2500 à 3500 tonnes entre 1995 et 2005 (Moujanni et al., 2017). La production paraît stable jusqu'en 2017, année où le ministère évalue la récolte marocaine au-delà de $6000 \mathrm{t}$ avec un cheptel qui aurait triplé à plus de 700000 ruches (Fellah-Trade, 2018; FIMAP, 2018). Les 900 millions de dirhams ( 84 millions $€$ ) mis en jeu par l'État depuis 2011 ont permis une diffusion massive de ruches modernes à cadres mobiles, désormais majoritaires, destinées à augmenter la productivité des ruches jusqu'alors largement

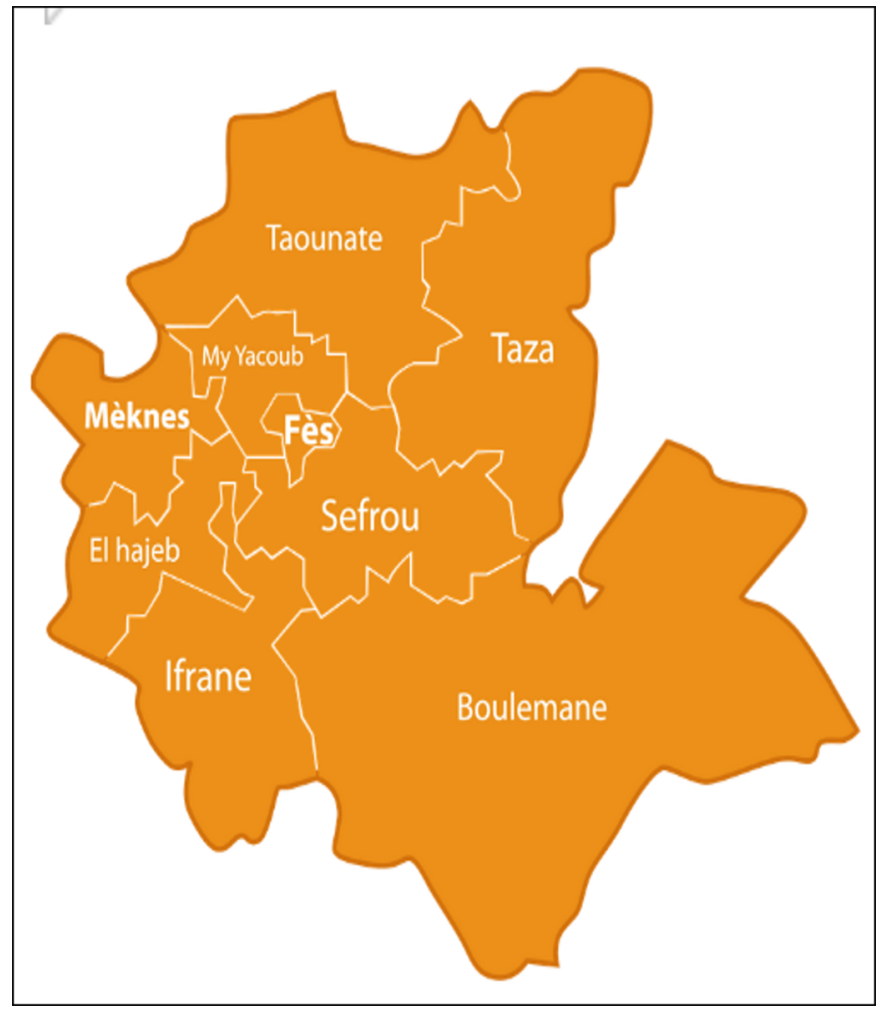

Fig. 1. Carte de la région Fès-Meknès.

Fig. 1. Map of the Fès-Meknès Region.

traditionnelles (Fig. 2 et 3 ). Les volumes ne sont pas confirmés par toutes les sources, mais celles-ci enregistrent une forte hausse du nombre d'apiculteurs dans le pays, de 23000 en 1996 à 32000 en 2013 (El Aïssi, 2016; Moujanni et al., 2017). Pour la région Fès-Meknès, près de 36 millions de dirhams (3,35 millions $€$ ) issus du PMV-Pilier-II ont concerné 12 projets apicoles, pour plus de 7000 ruches et 1923 apiculteurs (Bakhchou, 2017). En 2018, près de la moitié de la production de miel était concentrée dans les provinces de Taza et Tounate (Joual, 2019).

S'appuyant sur le succès d'expériences marocaines antérieures, la stratégie de développement agricole du PMV a défini comme centraux les processus d'agrégation des producteurs (Stührenberg, 2016), conduisant délibérément la politique régionale vers le soutien aux formes de production à dimension collective (associations, coopératives, etc.). Notre étude a été réalisée en concertation avec l'administration, afin de caractériser la composition des organisations professionnelles apicoles (OPA), qui sont essentiellement des coopératives, ainsi que les conduites techniques mises en place. Cette approche reste non exhaustive et ne permet pas d'établir des statistiques; mais elle fournit des indications capables de conforter les actions régionales sur la structuration de l'apiculture de Fès-Meknès, ou éventuellement de les rectifier.

L'activité apicole et son potentiel de développement sont fortement liés aux ressources mellifères de la région, connexe à la vie agricole et forestière des territoires. Ces ressources nécessitent un travail d'identification botanique permettant aussi d'évaluer les débouchés marchands. L'enjeu est d'établir les bonnes correspondances entre les miels produits et les ressources florales recherchées par les apiculteurs. 
Tableau 1. Effectif des OPA par province.

Table 1. Beekeeping groups number by province.

\begin{tabular}{|c|c|c|c|c|c|c|c|c|c|c|}
\hline Type d'OPA & Boulemane & El Hajeb & Fès & Ifrane & Meknès & Moulay Yacoub & Sefrou & Taounate & Taza & Total \\
\hline Coopératives & 111 & 13 & 12 & 17 & 25 & 13 & 44 & 34 & 130 & 399 \\
\hline Associations & 0 & 0 & 1 & 6 & 1 & 0 & 0 & 0 & 11 & 19 \\
\hline GIE & 0 & 2 & 0 & 0 & 1 & 0 & 0 & 0 & 3 & 6 \\
\hline Total OPA & 112 & 15 & 14 & 23 & 27 & 13 & 45 & 34 & 145 & 428 \\
\hline
\end{tabular}

\section{Matériels et méthodes}

L'enquête a été réalisée en 2017 en deux étapes successives, la première concerne la collecte des données institutionnelles auprès de la DRA, les DPA des 9 provinces ainsi que de l'ODCo (Office de développement et de coopération) à Rabat, Fès et Meknès. Les types d'organisations professionnelles (coopérative, association, GIE - groupement d'intérêt économique - ou union de coopératives), ainsi que les effectifs d'adhérents et de ruches, ont été répertoriés. L'étape suivante s'est déroulée auprès des OPA échantillonnées en concertation avec chaque DPA, sur le critère de leur représentativité et leur importance numérique dans la province, en incluant celles qui ne sont pas strictement apicoles. Au cours d'un entretien auprès des responsables de 2 à 9 organisations par province, nous avons réalisé 44 enquêtes, soit 7 à $45 \%$ des structures de chaque territoire. En amont de la production, nous avons répertorié les informations relatives à l'OPA elle-même, les pratiques apicoles, les ressources mellifères et les lieux de récolte. La lutte anti-varroa et l'élevage de reines sont les deux indicateurs de synthèse du niveau technique retenus. En aval, les données portent sur l'agrément d'autorisation des mielleries par l'ONSSA (Office national de la sécurité alimentaire), les équipements, le conditionnement et la commercialisation. L'analyse de correspondances multiples a été effectuée, avec le logiciel SPSS (IBM SPSS Statistics, v23), pour rechercher des associations entre les facteurs indicateurs d'actions de développement tels que la formation, le cheptel ou le matériel.

\section{Résultats}

\subsection{De très nombreuses coopératives apicoles}

Les OPA de la région sont plus nombreuses et comptent le plus grand nombre d'adhérents dans les provinces les plus étendues (Boulemane, Taza) et sont surtout des coopératives (Tab. 1). Une coopérative est à la fois une association et une entreprise où le pouvoir est exercé démocratiquement autour de valeurs d'ordre économique, social et culturel, indépendamment de la valorisation du capital investi. Les fonctions d'administrateur y sont gratuites et un directeur peut être désigné pour la gérer. Mais en dépit d'avantages fiscaux, l'une des principales difficultés des coopératives est la conception de fonctions managériales qui doivent concilier l'efficacité économique avec leur statut d'entreprise sociale au service de leurs adhérents (Oubal, 2016). D'autres OPA sont des associations mais qui ne peuvent pas englober des activités commerciales; d'autres encore sont des GIE, qui ont une vocation focalisée sur la commercialisation des produits sans considération éducative systématique. Toutes ces OPA peuvent rester indépendantes (Ifrane, Moulay Yacoub, Taounate) ou adhérer à des regroupements diversifiés plus rémunérateurs, comme la plupart de celles d'El Hajeb, Fès et Taza, formant alors des unions de coopératives, par exemple. De façon générale, les adhérents réalisent les travaux apicoles euxmêmes mais peuvent faire appel à des salariés pour les transhumances, la récolte ou les multiplications de colonies. Ces formes de production collectives sont des lieux d'émancipation pour les femmes vis-à-vis des tâches d'exécution que la société rurale traditionnelle leur assigne et certaines coopératives sont exclusivement féminines. Elles sont aussi un moyen efficace de permettre à des jeunes, diplômés ou non, de prendre part à l'économie locale en s'impliquant dans des conseils d'administration, donc des prises de décision. À titre indicatif, nous avons relevé que 35\% des administrateurs d'OPA ont un diplôme universitaire (Tab. 2).

\subsection{Un matériel de production modernisé mais des mielleries souvent vétustes}

Dans le groupe d'OPA étudiées, le nombre moyen de ruches à cadres (type Langstroth) est très disparate (Tab. 2); une coopérative atypique en possède 2600 (Ittihad, Ifrane). Les ruches traditionnelles ont été estimées lors des enquêtes à $6 \%$ du cheptel total; leur miel, extrait par pression, est vendu plus cher.

Le matériel de centrifugation est généralement vétuste ou partiel. Neuf coopératives disposent d'une miellerie agréée ONSSA aux normes d'hygiène strictes, qui autorise à vendre dans certains magasins ou salons. Les OPA disposent souvent d'un extracteur en inox individuel ou en commun, de capacité de 8 à 36 cadres. Mais $17 \%$ n'en possèdent pas et font alors appel au voisinage. Il ressort de nos enquêtes que ces apiculteurs comptent principalement sur les projets de développement pour financer ce matériel. 
Tableau 2. Caractéristiques des OPA apicoles par Province.

Table 2. Beekeepers associations' features by province.

\begin{tabular}{|c|c|c|c|c|c|c|c|c|c|c|}
\hline & Boulemane & El Hajeb & Fès & Ifrane & Meknès & Moulay Yacoub & Sefrou & Taounate & Taza & Total \\
\hline$\%$ jeunes & 13 & 20 & 30 & 31 & 48 & 20 & 45 & 21 & 35 & - \\
\hline $\mathrm{Nb}$ ruches & 2042 & 660 & 35 & 2832 & 420 & 190 & 650 & 945 & 780 & 8554 \\
\hline $\mathrm{Nb}$ moyen ruches/OPA & 255 & 220 & 17 & 708 & 140 & 95 & 216 & 189 & 156 & 1996 \\
\hline
\end{tabular}

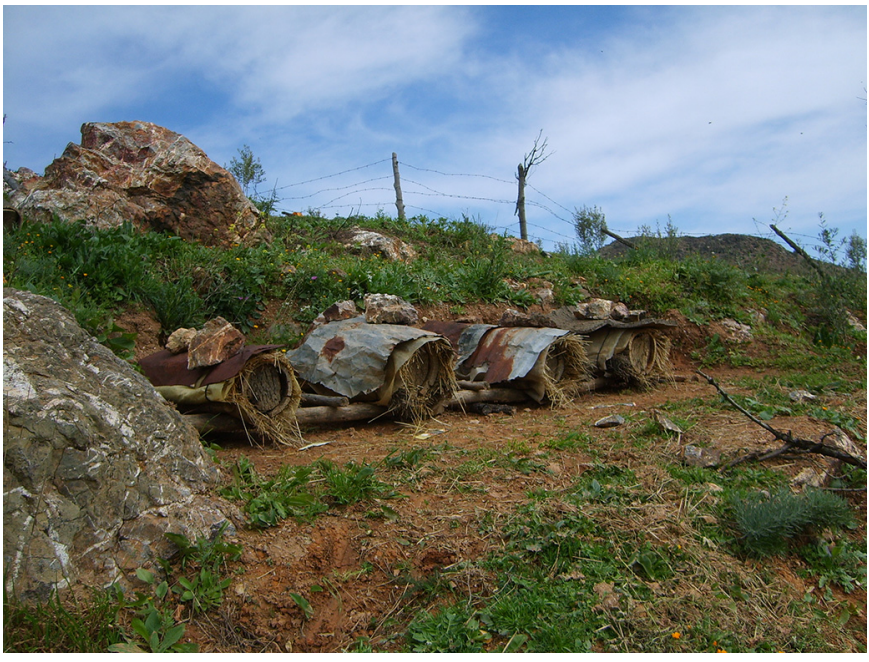

Fig. 2. Rucher de ruches traditionnelles.

Fig. 2. Apiary of traditional bee hives.

\subsection{Des techniques de production très diversifiées}

$90 \%$ des apiculteurs rencontrés prétendent élever la race d'abeille noire locale Apis mellifera intermissa: les autres disent utiliser la race saharienne A.m. sahariensis, vers Boulemane en particulier, mais un brassage important existe entre ces deux races principales. De fait, l'origine des abeilles n'est pas tracée lors des achats par l'État qui les attribue aux apiculteurs, si ce n'est qu'elle doit obligatoirement être marocaine. Le renouvellement du cheptel est partout étudiées, par division de colonies. L'élevage de reines avec greffage des larves est pratiqué dans $20 \%$ des structures étudiées, principalement à Boulemane et Taza. Nos résultats montrent que les apiculteurs de certains secteurs sont capables d'apprendre les techniques d'élevage de façon informelle, sans attendre d'accéder à des formations par un financement régional, contrairement aux achats de matériels de miellerie (Fig. 4a).

Le varroa n'est pas relevé comme préoccupation sanitaire dans 4 des 9 provinces et les apiculteurs ne traitent pas ou peu avec des médicaments; ils utilisent plutôt des plantes consumées dans un enfumoir, dont l'efficacité n'est pas renseignée. Notre analyse factorielle révèle que ceux ayant bénéficié de projets de développement, et donc de formations, utilisent plus fréquemment des médicaments homologués contre le varroa à base d'amitraze ou de fluvalinate (Fig. 4b).

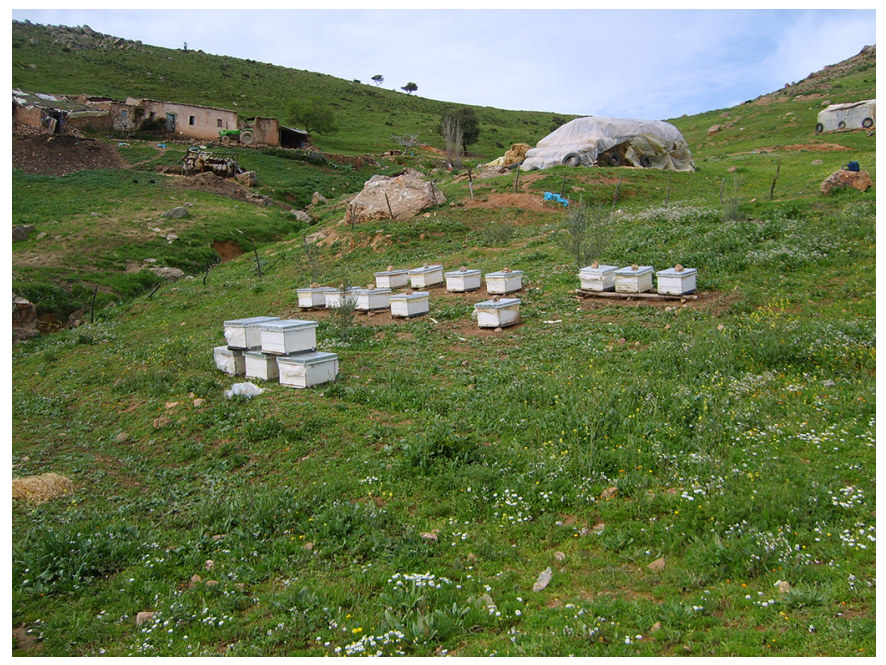

Fig. 3. Rucher de ruches Langstroth à proximité d'une habitation.

Fig. 3. Apiary of Langstroth bee hives nearby a dwelling.

\subsection{Du miel valorisé en circuit court}

La mise en pot à la récolte est généralisée à Boulemane, Fès, Ifrane, Meknès et Séfrou, sans stockage intermédiaire en fûts. Le miel est conditionné en bocaux neufs en verre ou en plastique de $1 \mathrm{~kg}, 500 \mathrm{~g}$ ou $250 \mathrm{~g}$, ou dans des pots et bouteilles de réutilisation censés véhiculer une image de miel véritable. La vente au détail est la plus commune, soit dans les locaux de la coopérative, comme à Boulemane et Séfrou, soit dans un réseau de magasins (Fès) ou dans les salons régionaux. $\mathrm{Au}$ moins $50 \%$ du miel est vendu lors des flux d'estivants et $30 \%$ lors des fêtes religieuses.

\subsection{Des ressources mellifères toute l'année}

Lors de ces enquêtes, nous avons inventorié les ressources mellifères de chaque province et élaboré un calendrier de floraison (Tab. 3). Les apiculteurs pratiquent des transhumances au gré de ces miellées, dans la région de Fès-Meknès (73\%) ou à l'extérieur, vers le Gharb ou Souss-Massa $(27 \%)$, habituellement au moyen de véhicules de location. Les miels produits sont multi-floraux ou mono-floraux comme le buplèvre (zendaz), le jujubier, le romarin, ou le caroubier (Tab. 4). S'il nous a été impossible de connaître la répartition de la production de miel régional par variété à l'occasion des enquêtes, nous disposons d'une étude de la DRA de 2010 

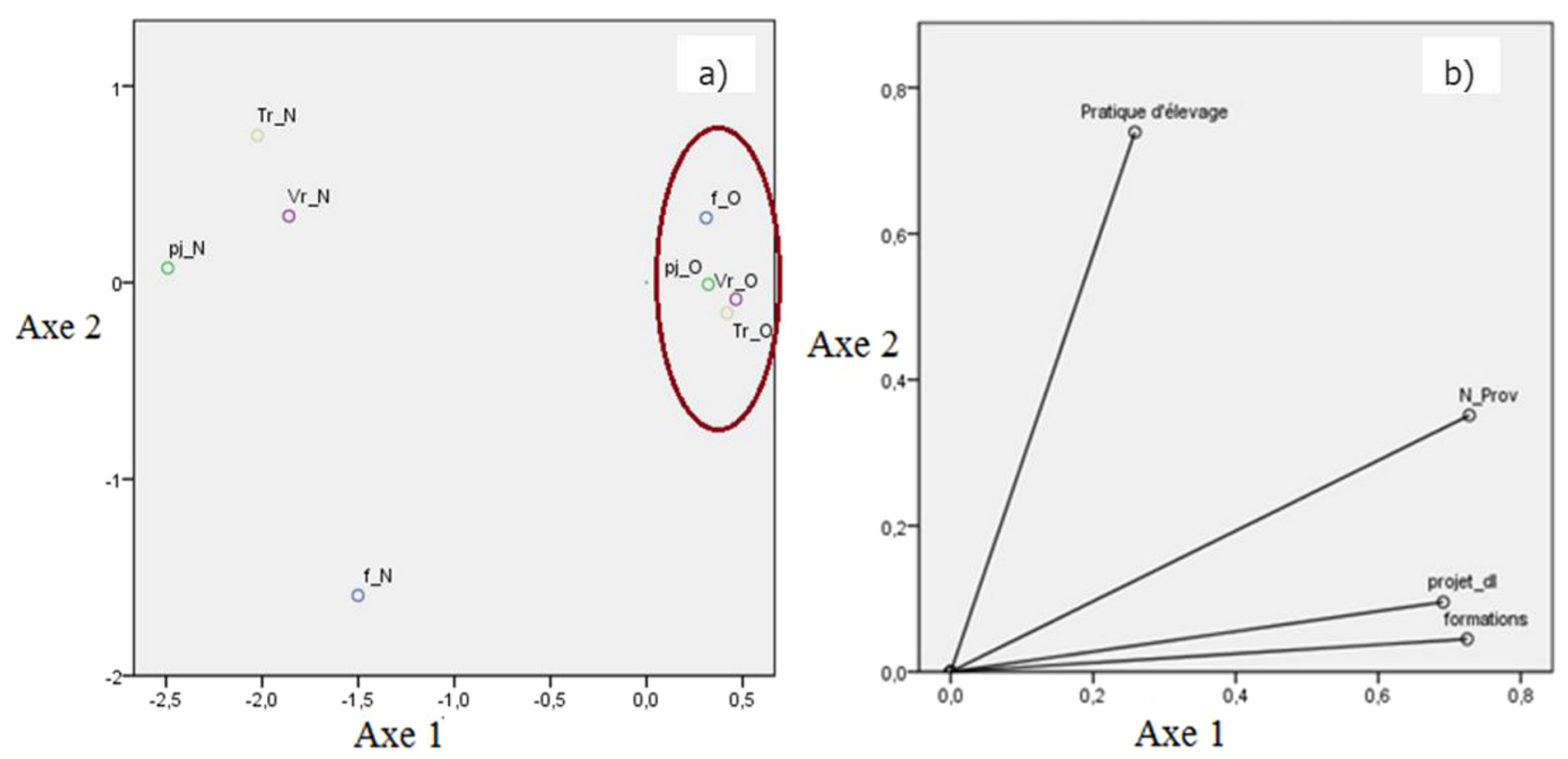

Fig. 4. Analyse multivariée des effets de la formation sur : a) l'élevage de reines ; b) facteurs sanitaires : formation : f_O/N : Oui/Non ; projet : pj_O/ $\mathrm{N}$ : Oui/Non; traitement varroa : Tr_O/N : Oui/Non; signalement varroa : Vr_O/N : Oui/Non; $\mathrm{n}^{\circ}$ province : N_Prov; pratique d'élevage : Oui/Non

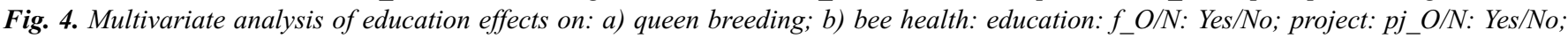
Varroa treatment: Tr_O/N: Yes/No; varroa reporting: Vr_O/N: Yes/No; $n^{\circ}$ province: N_Prov; breeding practices: Yes/No.

attestant, pour Fès-Boulemane, que le miel de romarin représentait près de la moitié de la production, suivi du miel de jujubier et de buplèvre (PCM Consulting, 2010).

\section{Discussion}

Malgré des difficultés d'ordre politique, technique ou climatique, l'apiculture marocaine bénéficie d'un accueil très positif dans le monde rural et son dynamisme se propage hors des frontières (Moujanni et al., 2017). C'est la première fois qu'une étude de cette envergure s'applique sur une région du Maroc, permettant de caractériser les formes collectives de production apicole alors qu'elles sont l'objet de soutiens publics. Au Maroc, les coopératives du domaine agricole (multipliées par 8 entre 2000 et 2013) sont de loin les plus nombreuses et la région de Fès-Meknès y est au premier rang, tout comme les adhérents diplômés y sont aussi en tête (Remacoop, 2016). Ces jeunes sont potentiellement capables d'interagir efficacement avec l'administration et les financements (Aroussi Bachiri et al., 2015). Une étude intergénérationnelle menée dans la Plaine du Saïss souligne le potentiel entrepreneurial des jeunes ruraux attachés à une agriculture familiale du terroir (Ameur et al., 2013), ce que confirme le succès croissant des aides auprès de jeunes créant des OPA (Bouzidi et al., 2015). L'investissement fort des femmes dans les structures apicoles de notre étude montre également l'intérêt de l'apiculture pour le développement rural.

Nous avons confirmé la persistance d'une apiculture traditionnelle dans la région, garante des savoirs ancestraux mais aussi d'une autosuffisance alimentaire. De fait, la situation hybridée d'une grande partie du cheptel marocain illustre la persistance d'une cohabitation des deux modes d'apiculture, y compris sur le territoire de l'étude (Mohssine et Daoudi, 1987; Moujanni et al., 2017).
L'un de nos résultats les plus marquants concerne la capacité des apiculteurs à pratiquer l'élevage de reines de façon autodidacte. La poursuite de formations organisées reste malgré tout nécessaire afin d'amorcer une vulgarisation en continu. L'élevage de reines s'avère économiquement rentable parce qu'il permet un gain de temps significatif pour le développement des colonies, même s'il est dépourvu de programme d'amélioration génétique. La mise en place d'un tel programme sous-entendrait une connaissance précise de l'état des lieux des races existantes et de leur distribution, alors que la seule couleur comme critère racial actuellement exigé ne garantit aucunement la traçabilité des races marocaines (Garnery et al., 1995). Dans des environnements distincts, un même génotype peut avoir des expressions phénotypiques différentes, ne reflétant pas exactement la diversité génétique de l'espèce. À l'inverse de certaines situations en Algérie (Adjlane et al., 2012), les pertes de colonies sont perçues comme peu alarmantes. La lutte contre le varroa, peu stricte, a alors recours à des phytothérapies empiriques peu onéreuses. La modeste productivité de l'apiculture affichée par les statistiques régionales semble traduire une faible technicité, probablement liée à ces deux lacunes dans les techniques apicoles performantes que sont l'élevage de reines et la lutte contre le varroa, qui sont directement liées à la question sensible de la mortalité. Il se peut que la forte mortalité soit moins durement ressentie du fait de la facilité de réapprovisionnement par l'État, et en ce sens cette mesure de réapprovisionnement peut être considérée comme un frein à l'innovation technique.

Nos résultats soulignent le rôle déterminant des aides publiques dans le processus d'équipement des mielleries. On note que la commercialisation du miel ne semble pas rencontrer de difficultés majeures pour les structures qui se sont organisées. À l'inverse, l'agriculture villageoise enclavée 
Tableau 3. Calendrier de floraisons dans la région. Les provinces sont désignées par les numéros ci-après : Boulemane (1); El Hajeb (2); Fès (3); Ifrane (4); Meknès (5); Moulay Yacoub (6); Séfrou (7); Taounate (8); Taza (9).

Table 3. Flowering calendar in the region.

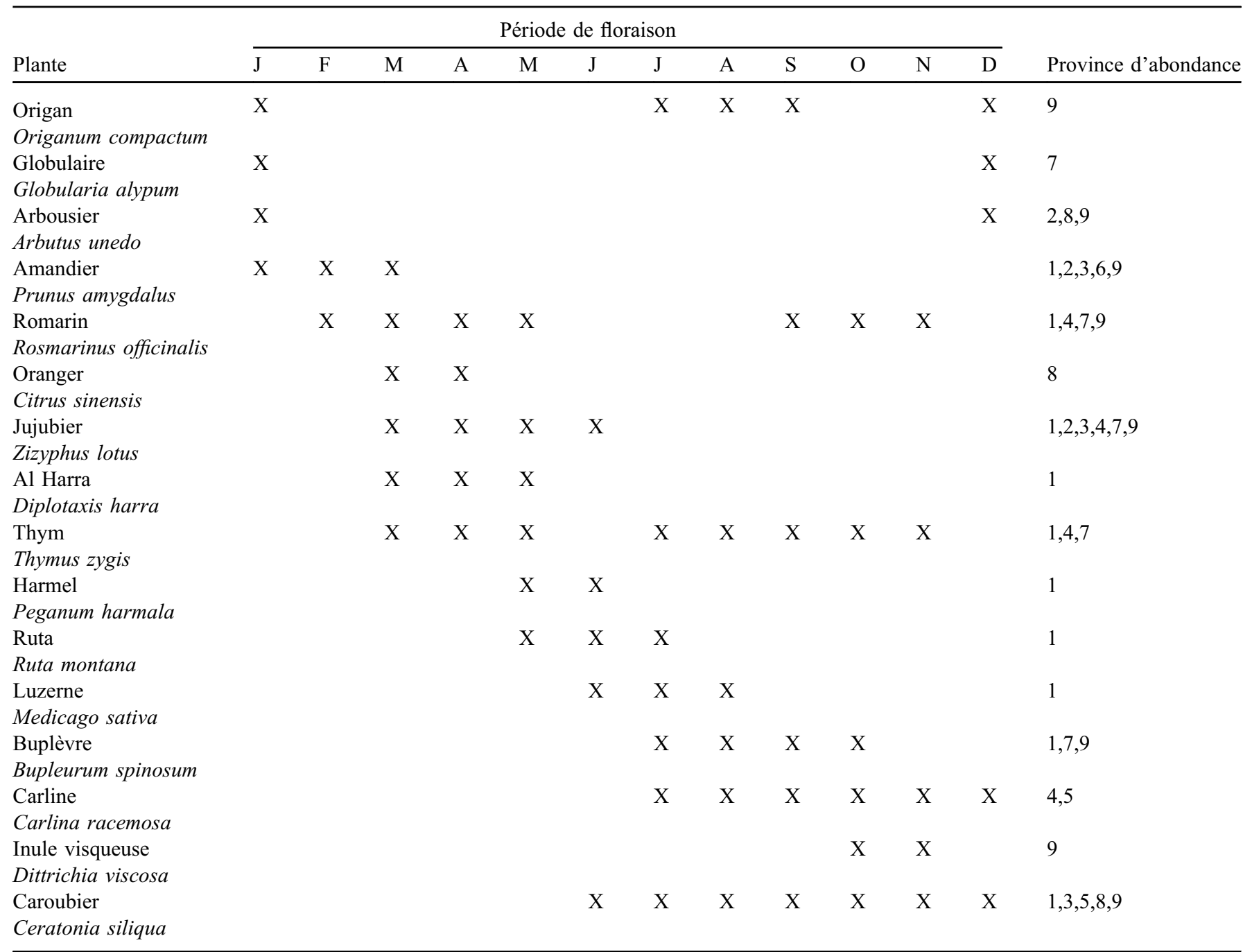

Tableau 4. Miels mono-floraux et multi-fleurs.

Table 4. Mono-floral and multi-flower honeys in Fès-Meknès Region.

\begin{tabular}{|c|c|c|}
\hline Type de miel & Composition florale & Provinces \\
\hline Caroubier & Ceratonia siliqua & $1,2,9$ \\
\hline Origan & Origanum sp & $1,7,9$ \\
\hline Romarin & Rosmarinus officinalis & $1,7,9$ \\
\hline Thym & Thymus zygis & $1,4,7,9$ \\
\hline Multi-fleurs de la forêt Oudka & $\begin{array}{l}\text { Arbutus unedo, Ridolfia segetum, Ammi visnaga, Morus alba, Lavandula } \\
\text { multifida, Matricaria chamomilla, Quercus rotundifolia }\end{array}$ & 8 \\
\hline Multi-fleurs & Ziziphora lotus, Carlina racemosa et autres plantes épineuses & 2 \\
\hline Multi-fleurs & $\begin{array}{l}\text { Ziziphora hispanica, Rosmarinus officinalis, Ziziphus lotus, Capparis spinosa, } \\
\text { Ammi visnaga }\end{array}$ & 3 \\
\hline
\end{tabular}


connaît des contraintes de mobilité généralement plus fortes, liées à l'accès aux transports en particulier, essentiel à l'activité marchande (Tazi, 2018). La participation à des salons est utile, car elle contribue à véhiculer l'image régionale d'un produit de terroir. L'utilisation d'emballages réutilisés, associée à l'authenticité du produit, peut être un atout pour le producteur mais reste un frein à la garantie d'une parfaite qualité du miel auquel se heurte 1'ONSSA. En outre, ces emballages introduisent un flou entre le miel issu des ruches modernes et traditionnelles, peu compatible avec une démarche qualité de terroir.

Outre les questions de qualité, la labellisation des miels requiert une perception favorable activant la réactivité des consommateurs, un partenariat public-privé identifiable, ainsi qu'une unité de la part des apiculteurs (Moujanni et al., 2017). Un cas de miel labellisé existe déjà dans la région : le miel de zendaz du massif de Bouiblane, qui rejoint six autres miels marocains ainsi démarqués (MAPM, 2018).

Une progression de l'apiculture transhumante, dite pastorale, serait favorable à la filière, à condition que la quantité des ressources mellifères disponibles soit évaluée convenablement et que les densités de ruches transhumantes puissent être raisonnées pour préserver les autres pollinisateurs d'une compétition dévastatrice. Une réflexion de la gestion du territoire et des ressources florales, concernant aussi bien la flore spontanée que cultivée, doit alors être menée en concertation avec les administrations de l'agriculture et de la forêt. Ces ressources mellifères représentent un bien commun limité pour lequel les pratiques des apiculteurs ne sont pas indépendantes les unes des autres. Des mises en situation expérimentale impliquant les acteurs peuvent faire émerger de nouveaux systèmes de cultures innovants et plus durables (Ameur et al., 2013). En apiculture, la transhumance et la gestion d'un assolement raisonné sont déjà traditionnelles dans certaines régions. Adaptant l'abeille au territoire autant que le territoire à l'abeille de façon systémique à l'issue de très longs processus, un exemple d'apiculture dans le sud marocain est organisé autour d'un système agraire assurant des floraisons sur 4 phénophases de la colonie d'abeille; il comprend des falaises arborées, des élevages avec figuiers de barbarie, des céréales propices aux adventices et enfin des pentes d'arganiers (Simenel et al., 2015). La forêt marocaine, propriété de l'État, est le siège d'un cortège de biens communs tels que les ressources mellifères et l'ensemble des pollinisateurs, mais aussi l'usage des espaces forestiers pour la production, l'habitat, les besoins domestiques ou les loisirs (Blérot et Mhirit, 1999). L'absence de prise en considération ou d'évaluation du potentiel mellifère entraîne actuellement des concentrations de colonies dans les mêmes secteurs, provoquant ainsi une compétition entre ruches et donc entre apiculteurs. On peut alors supposer un effet non négligeable dans les phénomènes de mortalité à moyen terme. Les densités élevées de ruches dans un contexte de ressources insuffisantes ont également été relevées par d'autres observateurs (Nicollet, 2018). Il est alors essentiel que les transhumances reposent sur la connaissance des communautés de végétation et du besoin global des pollinisateurs déjà en place, incluant l'évaluation du potentiel mellifère des espèces (Ion et al., 2018). Notre étude a pu distinguer l'ensemble des plantes mellifères de la région et mettre en évidence que des floraisons existent du mois de janvier jusqu'au mois de décembre. La cartographie des massifs de végétation et des quantités de ressources disponibles par secteur correspondra à la phase suivante de ce même projet, afin de la confronter aux productions de miels de la région; elle fera l'objet d'une restitution future.

Le Maroc n'atteindra pas son objectif de production en 2020, mais l'apiculture peut contribuer à une reprise en main des ruraux sur leur territoire, et à redonner un sens culturel à des pratiques anciennes tout en y intégrant des techniques modernes, ainsi qu'à valoriser des produits authentiques de qualité. Ces huit années de bilan pour la DRA permettent d'envisager une seconde étape de développement vers un processus régional d'identification des miels. L'effort devra rester soutenu sur l'accompagnement des structures collectives organisées dans les domaines de la commercialisation, de la gestion et du contrôle des ressources mellifères. On peut en attendre en particulier une autonomie renforcée pour les futurs investissements locaux, sans recours systématique aux subventions. Alors que la vulgarisation technique parait pour partie indépendante des formations ou des projets de développement, un cheptel régulièrement renouvelé sans intervention des collectivités de la région sera le signe d'une réelle appropriation de l'élevage en apiculture.

Remerciements. Nous tenons à remercier les administrations et l'ensemble des apiculteurs qui ont accepté de contribuer à cette enquête ainsi que le programme MCRDV du Ministère de l'Agriculture et de la Pêche Maritime du Développement Rural et des Eaux et Forêts qui a financé cette étude. Nos sincères remerciements aux relecteurs de la revue qui ont permis une plus grande clarté dans ce texte.

\section{Références}

Aazza S, Lyoussi B, Antunes D, Miguel MG. 2014. Physicochemical characterization and antioxidant activity of 17 commercial Moroccan honeys. International Journal of Food Sciences and Nutrition 65(4): 449-457. DOI: 10.3109/09637486.2013.873888.

Adjlane N, Doumandji SE, Haddad N. 2012. Situation de l'apiculture en Algérie: facteurs menaçant la survie des colonies d'abeilles locales A. m. intermissa. Cahiers Agricultures 21(4): 235-241. DOI: 10.1684 /agr.2012.0566.

Ameur F, Hamamouche MF, Kuper M, Benouniche M. 2013. La domestication d'une innovation technique: la diffusion de l'irrigation au goutte-à-goutte dans deux douars au Maroc. Cahiers Agricultures 22(4): 311-318. DOI: 10.1684/agr.2013.0644.

Aroussi Bachiri N, Faysse N, Abdellaoui EH, Sebgui M. 2015. Le retour des jeunes diplômés en zone rurale au Maroc: Quelles interactions entre trajectoires individuelles et projets collectifs? Alternatives Rurales (HS Jeunes ruraux): 37-47. Disponible sur http://alternatives-rurales.org/le-retour-des-jeunes-diplomes-enzone-rurale-au-maroc-quelles-interactions-entre-trajectoires-indivi duelles-et-projets-collectifs/.

Bakhchou S. 2017. Contribution à la caractérisation de la filière apicole dans la région Fès-Meknès. Ingénieur Agronomie, IAV Hassan II, 119 p.

Blérot P, Mhirit O. 1999. Le grand livre de la forêt marocaine. Sprimont (Belgique): Éditions Mardaga, 280 p. Disponible sur https://books.google.fr/books?id=v18t8Plo1YMC\&printsec $=$ front cover \&hl $=$ fr\&source $=g b s \_g e \_s u m m a r y \_r \& c a d=0 \# v=$ onepa ge\&q\&f=false.

Bouzidi Z, Kuper M, Faysse N, Billaud JP. 2015. Mobiliser des ressources techniques et sociales pour s'installer: stratégies des 
jeunes ruraux au Maroc. Cahiers Agricultures 24(6): 420-427. DOI: 10.1684/agr.2015.0781.

Chakir A, Romane A, Marcazzan GL, Ferrazzi P. 2016. Physicochemical properties of some honeys produced from different plants in Morocco. Arabian Journal of Chemistry 9: S946-S954. DOI: 10.1016/j.arabjc.2011.10.013.

Chambre-Agriculture. 2019. Les filières végétales phares de la région. Chambre d'Agriculture Région Fès-Meknès.

El Aïssi N. 2016. L'apiculture encore loin des objectifs du contratprogramme. L'économiste 4795. Disponible sur https://www. leconomiste.com/article/998916-1-apiculture-encore-loin-desobjectifs-du-contrat-programme [accessed 24/05/2019].

Fellah-Trade. 2018. Les chiffres clés de la filière apiculture. Disponible sur www.fellah-trade.com.

FIMAP. 2018. Contrat-programme de la filière apicole. Bilan 20112018. Rabat (Maroc): Fédération interprofessionnelle marocaine de l'apiculture, $33 \mathrm{p}$.

Garnery L, Mosshine E, Oldroyd B, Cornuet J. 1995. Mitochondrial DNA variation in Moroccan and Spanish honey bee populations. Molecular Ecology 4(4): 465-472.

Ion N, Odoux J-F, Vaissière BE. 2018. Melliferous potential of weedy herbaceous plants in the crop fields of Romania from 1949 to 2012. Journal of Apicultural Science 62(2): 1-17. DOI: 10.2478/jas2018-0017.

Joual S. 2019. Caractérisation pollinique et physico-chimique de la diversité de miels et plantes mellifères associées dans la région FèsMeknès. Ingénieur Agronomie IAV Hassan II, 238 p.

Kabbaj J. 2008. L'élevage moderne sept fois plus productif. L'économiste 2689. Disponible sur https://leconomiste.com/arti cle/l-elevage-moderne-sept-fois-plus-productif [accessed 24/05/ 2019].

Louveaux J, Abed L. 1984. Les miels d'Afrique du Nord et leur spectre pollinique. Apidologie 15(2): 145-170.

MAPM. 2015. Contrats Programmes pour le développement des filières de production. Rabat (Maroc): Ministère de l'Agriculture, de la Pêche Maritime, du Développement rural et des Eaux et Forêts, Département de l'Agriculture, 15 p. Disponible sur http:// www.agriculture.gov.ma/sites/default/files/contrats_program mes_vf.pdf.
MAPM. 2018. Les produits labellisés au Maroc. Rabat (Maroc): Ministère de l'Agriculture, de la Pêche Maritime, du Développement rural et des Eaux et Forêts, Direction de Développement des Filières de Production (DDFP), 70 p. Disponible sur http://www. agriculture.gov.ma/sites/default/files/produitslabellises.pdf.

Mohssine EH, Daoudi A. 1987. Discrimination et caractérisation des populations d'abeilles du Maroc. Ingénieur d'Etat en Zoologie. Rabat, IAV Hassan II, 86 p.

Moujanni A, Essamadi AK, Terrab A. 2017. L'apiculture au Maroc : focus sur la production de miel. International Journal of Innovation and Applied Studies 20(1): 52.

Nicollet B. 2018. L'Apiculture au Maroc: le miel du Maroc. Disponible sur https://abeille-et-nature.com.

Oubal K. 2016. Particularités des coopératives: vers la nécessité de conception des fonctions managériales spécifiques. Revue marocaine de recherche en management et marketing 1(13): 20.

PCM Project Cycle Management Consulting. 2010. Réalisation d'une étude sur l'identification et le développement des produits de terroir dans la région de Fès-Boulemane, $\mathrm{n}^{\mathrm{o}}$ 01/2010/DRA/Fès-Boulemane. Maroc: MAPM, $133 \mathrm{p}$.

Petretto GL, Tuberoso CIG, Fenu MA, Rourke JP, Belhaj O, Pintore G. 2017. Antioxidant activity, color chromaticity coordinates, and chemical characterization of monofloral honeys from Morocco. International Journal of Food Properties 20(9): 2016-2027. DOI: 10.1080/10942912.2016.1230745.

Remacoop. 2016. Statistiques. Revue marocaine des coopératives 6: 120.

Simenel R, Adam A, Crousilles A, Amzil L, Aumeeruddy-Thomas Y. 2015. La domestication de l'abeille par le territoire. Un exemple d'apiculture holiste dans le sud marocain. Techniques \& Culture 63 (1): 258-279.

Stührenberg L. 2016. Plan Maroc Vert: les grands principes et avancées de la stratégie agricole marocaine. Bulletins de synthèse souveraineté alimentaire 20. Disponible sur http://www.interreseaux.org/IMG/pdf/bds_no20_plan_maroc_vert.pdf [accessed 31/05/2019].

Tazi AG. 2018. Les coopératives agricoles au Maroc, un levier de développement miné. Les clés du Moyen-Orient. Disponible sur www.lesclesdumoyenorient.com.

Citation de l'article : Mohssine EH, Bakhchou S, Odoux J-F. 2020. Les organisations professionnelles apicoles dans la région de FèsMeknès au Maroc. Cah. Agric. 29: 12. 